\title{
BESSY II FEEDBACK SYSTEMS *
}

\author{
$\underline{\text { S. Khan }}^{\dagger}$, T. Knuth, BESSY, Berlin, Germany
}

\section{Abstract}

The present status of feedback systems to counteract unwanted beam motion and coupled-bunch beam instabilities in the BESSY II storage ring is reviewed.

\section{INTRODUCTION}

BESSY II [1] is a high-brillance synchrotron radiation source in Berlin/Adlershof. The first beam was stored in April 1998, regular user shifts started in January 1999.

Several effects tend to reduce the brillance of the synchrotron radiation delivered to the users:

- Unwanted motion of the closed orbit e.g. from ground vibration or from power supply ripple may increase the effective beam emittance seen by the experimenter.

- Longitudinal coupled-bunch oscillations caused by higher-order modes (HOMs) of the rf cavities may limit the beam current, deteriorate undulator spectra, and increase the beam size in dispersive regions.

- Transverse coupled-bunch instabilities from HOMs and due to the resistive-wall effect may also limit the beam current and increase the effective emittance.

Plans to counteract these effects by feedback systems are reviewed in this paper. For each project, the layout of the system and its expected performance are described, and the present status is reported.

\section{LOCAL ORBIT FEEDBACK SYSTEM}

Slow orbit drifts $(<1 \mathrm{~Hz})$ can be handled by the global orbit correction system of the storage ring using corrector coils integrated in the sextupole and dipole magnets. To counteract orbit motion in the $1-100 \mathrm{~Hz}$ regime on the $1 \mu \mathrm{m}$ level, the rate of sampling and correcting the beam should be of the order of $1 \mathrm{kHz}$, and dedicated computer hardware and magnets are required. As a prototype, a local feedback system with a closed bump enclosing an undulator (U-49) will be installed and tested in summer 1999. For a review of local and global correction schemes see e.g. [2].

As outlined previously [3, 4], the feedback system uses 4 window frame magnets, each equipped with horizontal and vertical coils on a yoke of granulated iron. Their design is a compromise between low inductance, low eddy currents, space requirements and sufficient kick strength. Excited by bipolar 20V/20A power supplies of $1 \mathrm{kHz}$ bandwidth, each corrector magnet produces a maximum kick of $0.9 \mathrm{mrad}$ horizontally and $0.7 \mathrm{mrad}$ vertically (at a beam energy of

\footnotetext{
${ }^{*}$ This work is funded by the Bundesministerium für Bildung, Wissenschaft, Forschung und Technologie and by the Land Berlin.

† Email: khan@bii.bessy.de
}

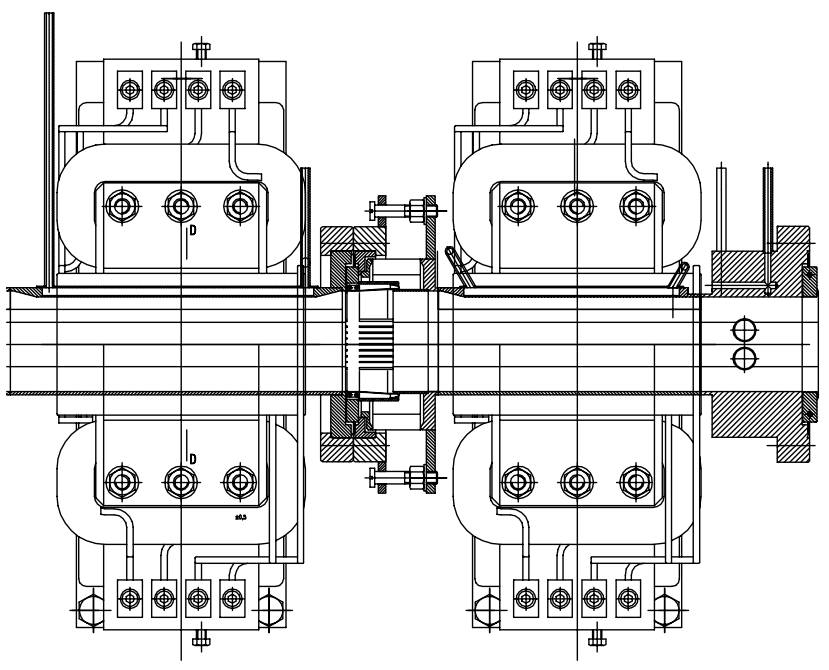

Figure 1: Vacuum chamber and corrector magnets to be installed on either end of the undulator chamber. The drawing shows the upstream end (beam from left to right).

1.7 GeV). The distance between the magnets, $255 \mathrm{~mm}$ from center to center, defines the dynamic range for displacing the beam to be $\pm 0.23 \mathrm{~mm}$ horizontally and $\pm 0.18 \mathrm{~mm}$ vertically.

Figure 1 shows the magnets and the vacuum chamber to be installed on either side of the undulator. The chamber is tapered from an octagonal cross section $(65 \times 35 \mathrm{~mm})$ to the elliptical profile $(60 \times 16 \mathrm{~mm})$ of the adjacent undulator chamber, and a bellow is placed between the two magnets. Beam position monitors (BPMs) on both sides of the undulator yield the electron beam position and angle within the closed bump.

In addition to the electron beam position, the information from photon BPMs is available. By rejecting photoelectrons below a tunable threshold, the background radiation from the adjacent dipoles can be suppressed [5].

The hardware (vacuum chamber, magnets, power supplies) has been delivered and awaits installation in the next suitable shutdown period. Software development is underway. Tests performed using Motorola MVME-162 CPUs under VxWorks were satisfactory. However, to allow for more flexibility, the use of VME-based DSPs is considered, while a VME master CPU would establish the communication with the BESSY II control system. 


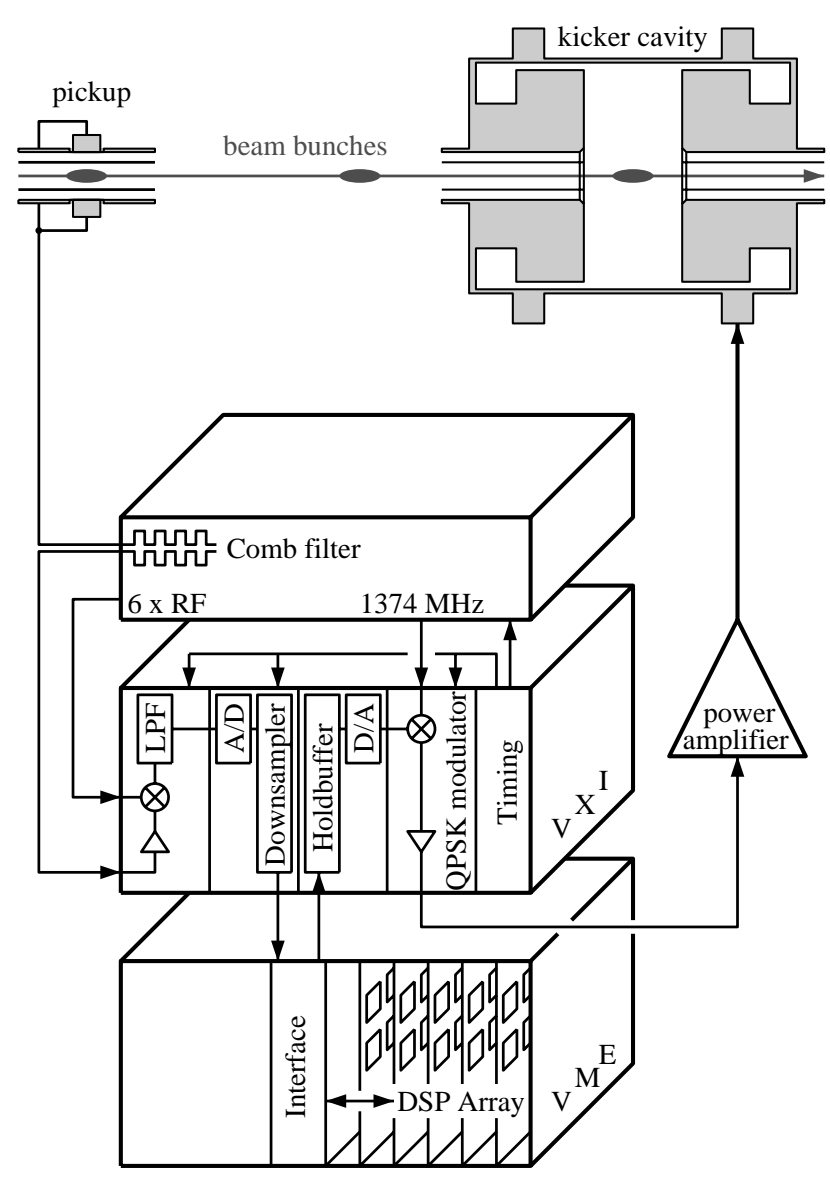

Figure 2: Layout of the longitudinal feedback system

\section{LONGITUDINAL BUNCH-BY-BUNCH FEEDBACK SYSTEM}

The BESSY II storage ring is operated using four DORIStype pillbox cavities with a rich HOM spectrum. Damping antennas have been added to suppress the most prominent HOMs [6], and tuning the HOM positions by controlling the cavity temperature is in preparation. Furthermore, the design of Landau cavities to further suppress coupledbunch instabilities and to increase the bunch length is underway. In addition to these measures, a longitudinal feedback system (LFB) will be installed in summer 1999.

The LFB electronics developed for the ALS (Berkeley), PEP-II (Stanford) and DA $\Phi$ NE (Frascati) [7, 8] will be used in combination with a kicker cavity designed for DA $\Phi N E$ [9] and modified for a bunch frequency of $500 \mathrm{MHz}$ [10]. For details on the kicker cavity see [11].

Figure 2 shows the LFB schematically. The bunch signal from a pickup passes a comb filter to produce a $3 \mathrm{GHz}$ signal for phase detection. The moment signal (phase-charge) is digitized at a rate of $500 \mathrm{MHz}$, downsampled and distributed to an array of DPSs, where a correction signal is computed. The D/A-converted correction signal QPSKmodulates a carrier at $1374 \mathrm{MHz}(11 / 4$ times the rf fre-

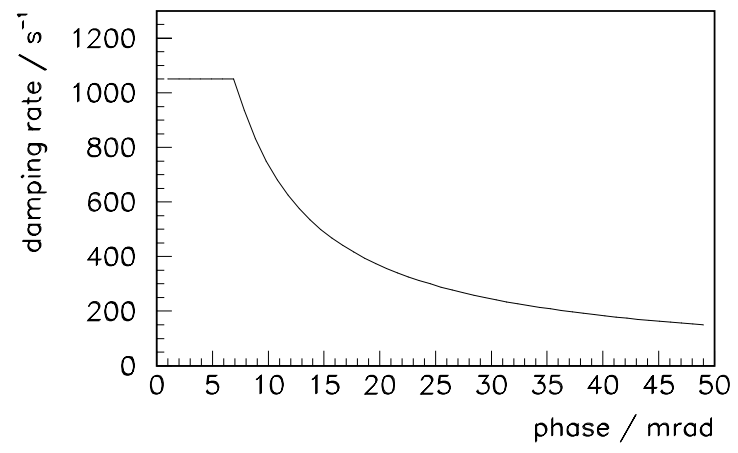

Figure 3: Long. damping rate versus phase deviation.

quency). A simulation demonstrates the beneficial effect of this particular modulation scheme [13].

The longitudinal damping rate can be expressed as [12]

$$
\frac{1}{\tau}=\frac{f_{\mathrm{rf}} \alpha}{2 \nu_{s} E / e} \cdot G,
$$

where $f_{\mathrm{rf}}$ is the rf frequency, $\alpha$ is the momentum compaction factor, $\nu_{s}$ is the synchrotron tune, and $E$ is the beam energy. The feedback gain i.e. the kick voltage per unit phase deviation $G=\Delta U / \Delta \phi$ is limited by the phase resolution and by the maximum amplifier power $P=220 \mathrm{~W}$ via $\Delta U \leq \sqrt{2 R_{s} P}$, where $R_{s}=960 \Omega$ is the kicker shunt impedance. Assuming 50\% power loss and saturation of the amplifier at $\Delta \phi=7 \mathrm{mrad}$ as an example, the maximum damping rate is about $1000 \mathrm{~s}^{-1}$, as shown in figure 3 . If necessary, the damping rate can be improved by adding rf power, by using more kickers, or - subject to operational experience - by increasing the gain.

The LFB electronics is currently being tested at SLAC, Stanford. The kicker cavity as well as the amplifier and other commercially available components have been delivered.

\section{TRANSVERSE BUNCH-BY-BUNCH FEEDBACK SYSTEM}

Apart from the transverse impedance of HOMs, the resistive-wall impedance can be quite significant for a synchrotron light source with narrow undulator chambers. For BESSY II, transverse growth rates of the order of $10^{3} \mathrm{~s}^{-1}$ were estimated [14].

The transverse feedback system (TFB) for BESSY II is modelled after the analog system developed for the ALS [15]. Figure 4 shows a block diagram of the TFB, where signals from two sets of button-type pickups approximately $90^{\circ}$ apart in betatron phase are used. The moment signals (displacement-charge) are detected at $3 \mathrm{GHz}$, differenced, mixed down to baseband, and combined in proper proportion. For offset rejection, the correction signals from subsequent revolutions are subtracted. The resulting kicks are provided by stripline kickers driven by $150 \mathrm{~W}$ power amplifiers. A $180^{\circ}$ power splitter is used to drive opposite 


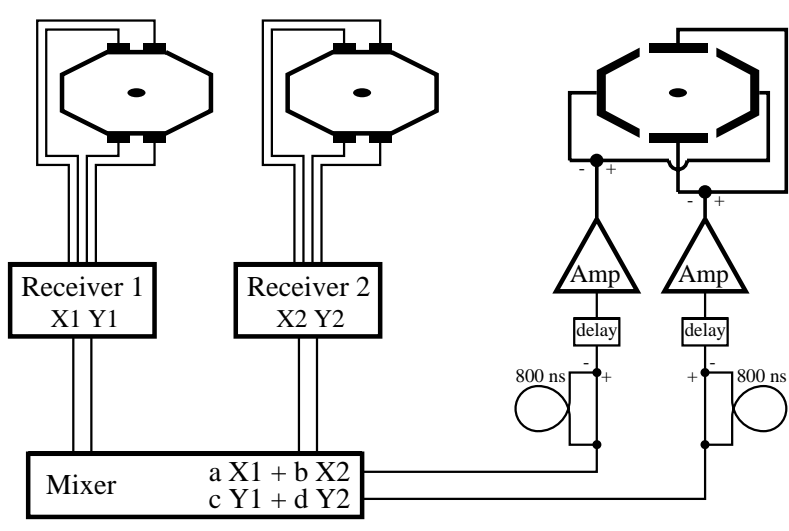

Figure 4: Block diagram of the transverse feedback system.

electrodes. Alternatively, the full power could be applied to a single electrode. The kicker geometry and performance is described in [11].

The damping rate of the TFB can be written as

$$
\frac{1}{\tau}=\frac{f_{\circ} \sqrt{\beta_{1} \beta_{2}}}{2 E / e} \cdot G,
$$

where $\beta_{1}$ and $\beta_{2}$ are the beta functions at the pickup and at the kicker position, respectively, and $G=\Delta U / \Delta y$ is the feedback gain i.e. the kick voltage per unit displacement.

With a $150 \mathrm{~W}$ amplifier connected to each pair of electrodes and assuming 50\% power loss, the vertical damping rate is shown in figure 5 at both ends of the relevant frequency range. In this example, the amplifier saturates at $\Delta y=1 \mathrm{~mm}$, leading to a maximum vertical damping rate of $5300 \mathrm{~s}^{-1}$ at low frequency, dropping to $3500 \mathrm{~s}^{-1}$ at $250 \mathrm{MHz}$. Due to the smaller shunt impedance in the horizontal plane (where the resistive-wall effect is irrelevant), the horizontal damping rates are $\sim 0.7$ times smaller.

The design of the kicker has been finalized, and its installation is scheduled for fall 1999. Power amplifiers have been purchased. The two receivers have been completed, the mixing chassis and the digital interface to the control system are underway.

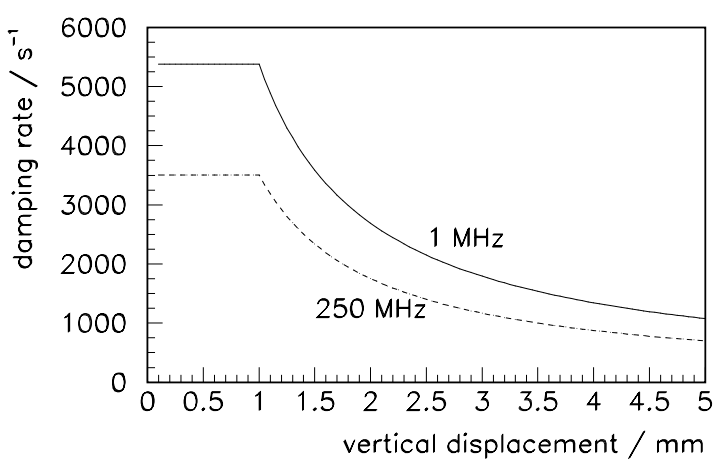

Figure 5: Transverse damping rate versus displacement.

\section{ACKNOWLEDGEMENTS}

The contributions of J. D. Gilpatrick (LANL, Los Alamos) to the local orbit feedback system are gratefully acknowledged. As for the bunch-by-bunch systems, valuable information and help was provided by W. Barry, J. Byrd, J. Corlett, G. Stover (ALS, Berkeley), J. Fox, H. Hindi, S. Prabhakar, D. Teytelman (SLAC, Stanford), A. Gallo, F. Marcellini, M. Serio and M. Zobov (INFN, Frascati).

\section{REFERENCES}

[1] R. Bakker for the BESSY II Project Team: 'Status and Commissioning Results of BESSY II', this conference.

[2] J. N. Galayda, Y. Chung, R. O. Hettel: in 'Synchrotron Radiation Sources - a Primer', ed. H. Winick (World Scientific 1994), p.344, and references therein.

[3] J. D. Gilpatrick, S. Khan, D. Krämer: 'A State-Variable Approach to the BESSY II Local Beam-Position-Feedback System', Proc. of the 5th Europ. Part. Acc. Conf., Sitges (1996), p.1902.

[4] S. Khan, D. Krämer, J. D. Gilpatrick: 'Layout and Timedomain Simulation of a Local Beam Postion Feedback System for BESSY II', Proc. of the 5th Europ. Part. Acc. Conf., Sitges (1996), p.1839.

[5] K. Holldack et al.: 'Suppression of Dipole Background from Position Signals of Photon BPMs', BESSY Jahresbericht 1995, 497.

[6] N. Lehnart, H. Petersen: 'Ferrit-Dämpfungsantennen gegen parasitäre Cavity-Modes in den DORISHohlraumresonatoren', DESY Techn. Notiz H2-77/12 (1977).

[7] D. Teytelman et al.: 'Operation and Performance of the PEP-II Prototype Longitudinal Damping System at the ALS', Proc. of the 1995 Part. Acc. Conf., Dallas (1995), p. 2420 .

[8] S. Prabhakar et al.: 'Commissioning Experience from PEPII HER Longitudinal Feedback', Proc. Beam Instrumentation Workshop, Stanford, AIP 451 (1998), p.529.

[9] R.Boni et al.: 'A Waveguide Overloaded Cavity as Longitudinal Kicker for the DA $\Phi$ NE Bunch-by-Bunch Feedback System', Part. Acc. Vol.52 (1996), p.95.

[10] A. Gallo et al.: 'Adaption of the DA $\Phi$ NE Longitudinal Kicker Geometry to the Needs of the BESSY-II Synchrotron Light Source', DA $\Phi$ NE Technical Note RF-21 (1998).

[11] T. Knuth, S. Khan: 'Longitudinal and Transverse Feedback Kickers for the BESSY II Storage Ring', this conference.

[12] M. Bassetti et al.: 'DA $\Phi$ NE Longitudinal Feedback', Proc. of the 3rd Europ. Part. Acc., Berlin (1992), p.807.

[13] S. Khan: 'Simulation of Longitudinal Coupled-Bunch Instabilities in BESSY II', Proc. of the 6th Europ. Part. Acc. Conf., Stockholm (1998), p.966.

[14] S. Khan: 'Simulation of Transverse Coupled-Bunch Instabilities', Proc. of the Part. Acc. Conf., Dallas (1995), p.3019.

[15] W. Barry et al.: 'Transverse Coupled-Bunch Feedback in the Advanced Light Source (ALS)', Proc. of the 4th Europ. Part. Acc. Conf., London (1994), p.122. 